\title{
Nursing teaching role performance from the viewpoint of discharging patient from the Abadan and Khorramshahr hospitals
}

\author{
Noorollah Tahery ${ }^{\mathrm{a}}$, Shirzadegan Razieh ${ }^{\mathrm{b}}$, Aghababaeian Hamidreza ${ }^{\mathrm{c}}$, Sahar Geravandi ${ }^{\mathrm{d}}$, \\ Mohammad Javad Mohammadi ${ }^{\mathrm{e}, *}$ \\ a Department of Nursing, Abadan School of Medical Sciences, Abadan, Iran \\ ${ }^{\mathrm{b}}$ Social Determinant of Health Research Center, Lorestan University of medical sciences, Khorramabad, Iran \\ ${ }^{\mathrm{c}}$ Department of Nursing \& Emergency Medicine, Dezful University of Medical Sciences, Dezful, Iran \\ ${ }^{\mathrm{d}}$ Asadabad School of Medical Sciences, Asadabad, Iran \\ ${ }^{\mathrm{e}}$ Department of Environmental Health Engineering, School of Public Health AND Environmental Technologies Research Center, Ahvaz Jundishapur University of Medical \\ Sciences, Ahvaz, Iran
}

A R T I C L E I N F O

\section{Keywords:}

Patient

Education

Nurses

Discharge

Iran

\begin{abstract}
A B S T R A C T
Introduction: Patient education is one of the most important roles of nurses and nurses' commitment to doing so is influenced by their understanding of their educational role.

Objective: The aim of this study was to determine the viewpoints of patients who are being discharged from Abadan and Khorramshahr hospitals regarding the amount of nursing education to the patient.

Materials and methods: This is a descriptive cross-sectional study in which 576 patients from all patients admitted to the internal and surgical wards. Sampling was done by Convenient Sampling method.

Results: From the patient's point of view, nursing education was carried out in this way, 52.7\% were desirable, $41.2 \%$ were on average and $6.1 \%$ unsatisfactorily. Between patients' viewpoints on patient education with the reason for referring patients to the hospital $(P<0.001)$, The gender of the patients $(p=0.03)$ and the admission ( $\mathrm{p}=0.03$ ) were statistically significant.

Conclusion: The results of the study showed that about half of the nurses had been trained to them, given the importance of educating the patient and its role in developing the culture of self-care, reducing hospitalization, reducing medical costs, and highlighting the social status Nurses, nursing managers seem to be more emphasis on this issue.
\end{abstract}

\section{Introduction}

Patient education is one of the main aspects of patient care and patient-centered process that is based on patient's needs and health-care team in helping him to participate in informed and informed decision making. ${ }^{1,2}$ It is considered as an important task and one of the quality standards for nursing care, ${ }^{3,4}$ and is one of the most important components of quality health care services. ${ }^{5}$ Education, especially in nursing, is a tense experience that is one of the tensest jobs known. ${ }^{6}$

Patient education improves patient satisfaction, improves quality of life, ensures continuity of care, relieves anxiety, reduces the incidence of disease, increases participation in health care programs, and empowers the patient to carry out daily life activities, early departure to bed, shorter stay in the hospital, going to a home-based recovery, in addition, significantly reduces the cost of care and treatment, with each dollar spent on patient education cost $\$ 3$ to $\$ 4$ in costs. ${ }^{7}$ The proliferation of chronic and debilitating illnesses and the inability to admit patients at a prolonged time due to the lack of hospital facilities, the cost of exorbitant exertion and the onset of economic pressures on the families of these patients, believing that prevention is better than treatment, and also the prevention of hospital infections is the all-inclusive provision of self-care for patients and their importance. ${ }^{8} \mathrm{Un}$ doubtedly, the lack of this necessary information from the patient leads to his inability to deal with the illness and its complications, the need to visit more health centers, and frequent hospitalization, the unnecessary occupancy of hospital beds needed by patients with a severe condition. More, the huge cost to the patient and the hospital, the financial and psychological and social problems for the patient's family after repeated admissions and ultimately the loss of medical and nursing care where it is not necessary. ${ }^{9}$

\footnotetext{
*Corresponding author. Tel.: + 989355439707; fax: + 986133332015 .

E-mail address: javad.sam200@gmail.com (M.J. Mohammadi).
} 
Patient education is defined as a planned learning experience that uses the combination of methods such as teaching, counseling and behavior modification techniques to influence the patient's health knowledge and behaviors. In fact, patient education is a dynamic and continuous process that takes into account the most basic human needs and activities from admission to discharge, and is one of the characteristics of nursing care quality for all patients in all aspects of treatment. ${ }^{10}$ For example, a study on elderly patient education that could have a significant impact on increasing compliance with treatment regimens and establishing appropriate communication between nurses and patients showed that $15 \mathrm{~min}$ of training to the patient improved $50 \%$ of dietary intake. ${ }^{11}$ Mohammad Pour (2006) states that $47.8 \%$ of nurses have a positive attitude towards patient education. ${ }^{12}$ The training provided by the patients themselves was $65.7 \%$ in a study and $40.45 \%$ in the other study and had about $10.2 \%$ severe educational need. And in these two studies, more than half of the participants in the study tended to have more information about their illness before going home. ${ }^{12,13}$ Nursing teaching role performance include: training at the time of discharge, dietary education, drug regime, in home patient activity, etc.

Considering that inadequate education of the patient as one of the important factors of re-hospitalization, post-discharge complications, delayed patient recovery, increased costs of treatment, increased hospital infections, etc. is in our country, ${ }^{11}$ awareness of this issue is also related to the language and views of the recipients of the service, namely, the patients who are the best source of confirmation or Denial of the implementation of this process can help authorities for better and patient-centered planning. Therefore, this study was conducted to determine the viewpoints of the patients being discharged from Abadan and Khorramshahr hospitals regarding the level of nursing education.

\section{Materials and Methods}

This research is a descriptive cross-sectional study. The population of this study was all patients admitted to the internal and surgical wards of the Abadan hospitals (Taleghani and Shahid Beheshti) and Khorramshahr (Valiasr Hospital) during the study period. To determine the sample size, the formula for estimating the ratio $\frac{Z_{1}^{2}-\frac{\alpha}{2} \times p(1-p)}{d^{2}}$ was used. That in this formula $\alpha=0 / 05, p=0 / 5$ and $d=0 / 1$ are considered. The sample size was calculated to be 96 people for each ward and in total (576 people were evaluated according to (6 wards research environment) four internal and surgical sections of men and women in Taleghani hospital and two internal and surgical sections in Valiasr Hospital). Sampling was done by Non-probable sampling method (purposive sampling technique) so that from the start of the study, all patients who had the criteria for entering the study were selected as samples and this work continued until the final sample size reached. The criteria for entering the study include: All patients who have been hospitalized for at least 2 days in the internal or surgical wards of the hospitals and have been issued a discharge order at the time of the study, lack of specific psychological problems, ability to speak, and desire to Participate in the study. Unwillingness to participate in the study was considered as an exit criterion.

Data were collected using a nursing patient teaching role questionnaire. ${ }^{14,15}$ The validity of the questionnaire was reviewed by faculty members and its reliability was assessed using Cronbach's alpha test (= $0.85)$.

The questionnaire includes 11 questions about the nature of the disease, 6 questions about the complications of the disease, 9 questions about drug regimen, 3 questions about the activity, 3 questions about the diet and 4 questions in the field of other Items are set. Patients based on received training from admission to discharge, full education options (so that there was no ambiguity left to the patient and considered the patient to be in need of education), partial training (there were uncertainties for the patient, and Patient expresses the need for further education), and chooses not to be educated. in addition, there is no option considered for all questions. The score a questionnaire includes: to answer the full training is score of 3 , to answer the relative teaching is score 2 , and for untrained is score 1 considered.

The minimum and maximum scores that each of the study units can receive from this questionnaire was 36-108. In the end, for data interpretation, those who scored $36-60$, poorly trained, those who scored 61 to 84 , were relatively well-trained, and those who achieved 85 to 108 had a very well trained was considered. The data from patients by a nursing expert that referred to the hospitals and through interviewing and asking questions about the questionnaire phrases and after completing the informed consent form were collected. To analyze the data were used of central indicators and dispersion for calculating the mean and frequency, and to determine the relationship between patient's viewpoints on patient education and their level of education, one-way analysis of variance, patients' gender from independent $\mathrm{T}$ test and the determination of the relationship patient's viewpoints on patient education and their ages the Pearson Correlation Coefficient was used. The significance level for the above tests was considered to be 0.05 . Data were analyzed using spss.16 statistical software.

\section{Ethical considerations}

The Ethics Committee of Ahvaz Jundishapur University of Medical Sciences approved the study protocol. This study was originally approved by the Research Council of Ahvaz Jundishapur University of Medical Sciences with code U-88247.

\section{Results}

The findings of this study showed that the mean age of patients was $37.5 \pm 18.3$ years, the average days of admission were $3.4 \pm 2.4$ days, $63.4 \%$ of the samples were female and the rest were male, $70.4 \%$ were urban and the rest were rural, $4 / 25 \%$ were illiterate, $40.4 \%$ had secondary education, $27.1 \%$ had a diploma and $7.1 \%$ had university education.

Regarding the reason for referring to the hospital, $21.1 \%$ of the problems were in the kidneys and ducts, $20.4 \%$ of women, $18.3 \%$ of digestive problems, $16.2 \%$ of orthopedists, and the rest were admitted to the hospital for other reasons. In terms of job, $62.7 \%$ were housewives, $22.7 \%$ were free-of-charge, $7.3 \%$ were employees and $5.5 \%$ were retired and $1.8 \%$ were unemployed. $56 \%$ of the surgery and the rest were hospitalized. The findings of this study also showed that only $52.7 \%$ of the patients believed that nurses had a good educational role (Fig. 1).

The results of the study showed that there is a significant relationship between the cause of referral of patients to the hospital and their viewpoint regarding patient education $(\mathrm{P}<0.001$ ) (Fig. 2).

Tukey's test showed that the highest difference was found between

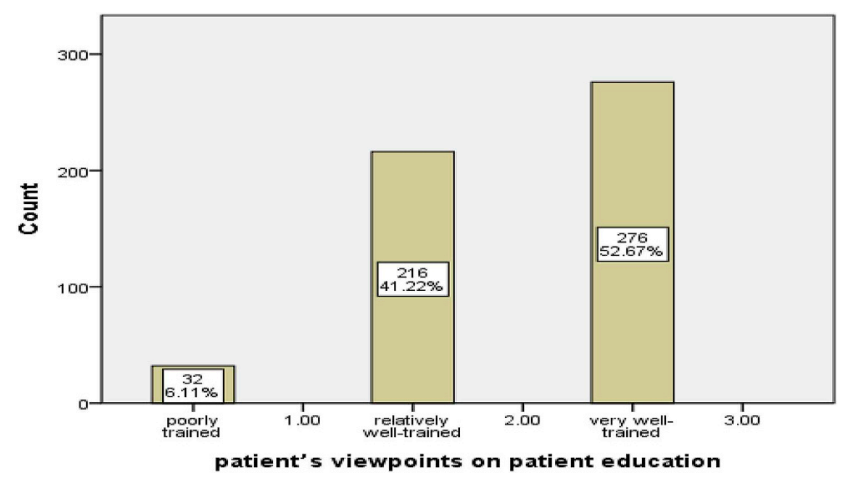

Fig. 1. Nurses' education to the patient from the point of view of discharged patients. 




Fig. 2. Relationship between gender and hospitalization of patients with their viewpoints on nursing education.

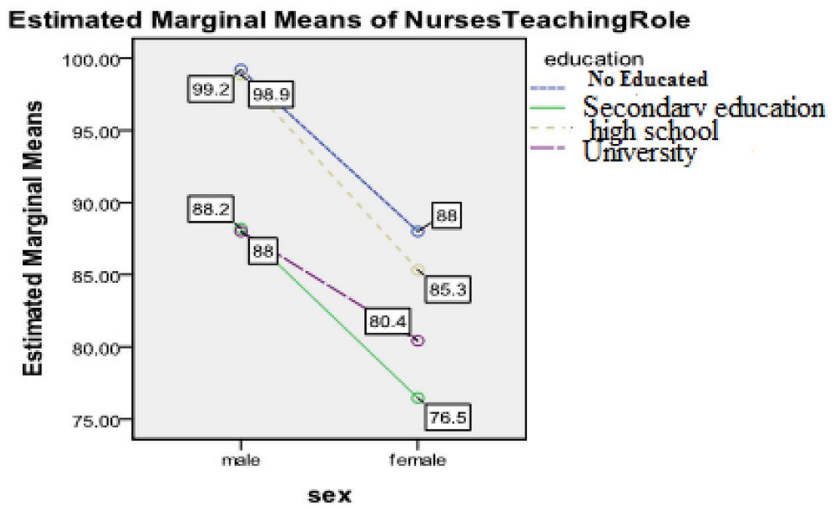

Fig. 3. Relationship between the level of education and the sex of patients with their viewpoints on nurse-patient education.

patients referred to orthopedic with women $(\mathrm{P}<0.001)$ and heart $(\mathrm{P}=0.03)$, women and gastrointestinal causes $(\mathrm{P}<0.001)$, women and kidney and vein diseases $(\mathrm{P}<0.001)$ (Fig. 2).

Two-way analysis of variance showed that there is a statistically significant relationship between sex and hospitalization of patients with their viewpoints on patient education by nurses $(\mathrm{p}=0.03)$.

According to one-way ANOVA, there was a significant relationship between the degree of education of patients and their viewpoints on nursing education. So, Tukey's test showed that the highest difference was observed between the patients with elementary education with those with university education $(\mathrm{p}<0.001)$ and those with secondary education ( $\mathrm{p}=0.001$ ) (Fig. 3 ).

The results of Pearson correlation coefficient indicated that there was no statistically significant relationship between the age of the patients and their viewpoints on nursing education $(r=-0.17 \mathrm{p}=0.7)$, also by independent $t$-test There was no statistically significant relationship between the place of residence of patients (urban and rural) and their viewpoint regarding nursing education $(\mathrm{p}=0.06)$.

\section{Discussion}

The results of the study indicate that almost half of the patients expressed that nurses did not fully fulfill their educational role, despite the fact that in many studies nurses believed that their education was important to the patient. ${ }^{3,9}$ But in practice, this fundamental role, which the researcher believes is appropriate to make a significant contribution to the nursing position in society, is not well implemented. Of course, nurses in various studies have also explained the reasons for the lack of proper implementation of this role. These include low numbers of nurses, high numbers of patients, high volumes of nurses' work and even denial of education from patients. ${ }^{16,17}$ It seems that nursing managers should take this nursing role more seriously and consider it as a fundamental measure in assessing nurses as well as selection of sample nurses in order to be able to promote nursing and lead nurses to their core roles in the future see progress. The results of the study showed that male patients more than women believed in the educational role of nurses, Marilyn et al. Stated that men are more concerned with teaching and receiving nurses than women, ${ }^{18}$ probably this has led to That men are more likely to seek solutions to their illness and ask nurses about their illness to improve the situation.

The study also found that people with lower education had lower grades than nurses, but those with higher education were expected to be more likely to learn about their health and disease, but the result was quite the opposite expected. Perhaps they felt that they had enough information about their disease or that nurses might have had such an impression about them that they had less to train them. Azimi et al., Mac and colleagues state that one of the barriers to educating patients is a small amount of nurses, and nurses are likely to be afraid of their ignorance in dealing with people with higher education, which usually has a lot of information about they have illnesses, they are less involved with education. ${ }^{16,19}$

Patients in the surgical ward (men and women) have a more positive attitude toward patient education than patients in the internal ward. Some studies on the difference in patient education by nurses in different hospitals and their educational level have the results ${ }^{20,21}$ but there has been no study on patient education in different parts or researchers have not had such an access.

Therefore, considering the differences in the volume of work in different parts and the type of hospitalized patients and the difference in the level of consciousness in patients in the internal and surgical wards, and in some cases, the difference in age and gender in certain patients, seems to be necessary in This is especially the case studies.

\section{Conclusion}

The results of this study showed that about half of nurses had been trained to patients from the perspective of patients. Therefore, it is suggested that nursing managers to better implement nursing education planning, such as holding training sessions on patient education for nurses, the effect of patient education on assessment of nurses, appreciation of nurses who train patients and so on take.

\section{Conflicts of interest}

There are no conflicts of interests among the authors.

\section{Acknowledgments}

This study was funded by Research Council of Ahvaz Jundishapur University of Medical Sciences (Grant No: U-88247). The authors are grateful to Deputy of Research Affairs of Ahvaz Jundishapur University of Medical Sciences for the financial support as well as the patients and nurses participating in this study.

\section{Appendix A. Supplementary data}

Supplementary data to this article can be found online at https:// doi.org/10.1016/j.cegh.2019.01.010.

\section{References}

1. Hasanzadeh F, Shamsoddini S, Moonaghi HK, Ebrahimzadeh S. A comparison of face to face and video-based education on attitude related to diet and fluids adherence in hemodialysis patients. Q Horiz Med Sci. 2011;17(3):34-43.

2. Helliwell PSIG. Ethnic differences in the use of prescription drugs: a cross-sectional analysis of linked survey and administrative data. Rheumatology. 2003;42(5):1197-1201

3. Tahery NRZM, Kaial M i, Rashidi M, Yaghoobi M, Shirzaei K, Koleini Z. Importance of Patient education according Abadan nursing students' opinions. J Birjand Univ Med 
Sci, Sch Nurs Midwifery. 2011;8(2):100-106

4. Mardani Hamuleh MSVA, Roozitalab M. Compare the importance and performance of patient education services in the view point of nurses and patients. $J$ Univ Med Sci Q. 2010;8(4):49-55

5. Yael LIP, Janna Sh. Barriers to patient education and their relationship to nurses' perceptions of patient education climate. J Clin Nurs Stud. 2017;5(4):65-72 2017;5(4):65-72

6. Tahery NKM, Yaghoobi M, Koleini Z. The stressors of clinical training in nursing students-Abadan nursing faculty. Mod Care Scient Q Birjand Nurs Midwifery Facul. 2009;8(3):159-165 1390.

7. Adib-Hajbaghery M, Zare M. The barriers to patient education from the viewpoint of nurses in iran: a systematic review. J Nurs Midwifery Urmia Univ Med Sci. 2017:15(7):544-558

8. Aghakhani N, Nia HS, Ranjbar H, Rahbar N, Beheshti Z. Nurses' attitude to patient education barriers in educational hospitals of Urmia University of Medical Sciences. Iran J Nurs Midwifery Res. 2012;17(1):12-15.

9. Marcum J, Ridenour M, Shaff G, Hammons M, Taylor M. A study of professional nurses' perceptions of patient education. J Cont Educ Nurs. 2002;33(3):112-118.

10. Mansoorghanaei RMS, Tabari R. Nurses viewpoints about facilitator and inhibitor factors of patients education. Res Med Educ. 2011;3(1):27-33.

11. Farouki Far MKF, MostafaZadeh M, Alijanpour S. The importance of patient education: the attitude of nurses in the hospitals of babol. Biannual medical education. Babol Univ Med Sci. 2014;3(1):59-65.

12. Mohammad Pour ADNN. MThe survey of the patient educational need on discharge phase in Gonabad health and treatment centers. Ofogh-e-Danesh J Gonabad Univ Med
Sci Health Serv. 2005;12(4):34-39

13. Rahmati Chagharvand MGRP. Patients' view on training programs during hospitalization in surgical wards of Shahid Mohammadi Hospital. Bandar Abbas Med $J$ Hormozgan Univ. 2006;5(4):27-38

14. Soltani Khabisi AAfM, Haghdost A, Mohammad Alizadeh S. Nurses' performance in training patients from the point of view of patients discharging from medical- surgical wards of hospitals affiliated to kerman University of Medical Sciences. J Med Educ Dev Cent. 2005;3(1):51-57.

15. Mobaraki AKZ. The study of nursing students attitudes towards patients TrainingYasouj University of Medical Sciences. Dena Q J Yasouj Facul Nurs Midwifery. 2005; 1(3):61-67

16. Vahedian Azimi AEF, Hedayat K. Barriers and facilitators of patient's education: nurses' perspectives. Iran J Med Educ. 2011;11(6):620-627.

17. Aziznejad PZA, Hossaini SJ, Bijani A. Nurses and nurse managers opinions about the patient's training barriers. J Babol Univ Med Sci. 2010;12(1):60-64.

18. Oermann $\mathrm{MH}$, Harris $\mathrm{CH}$, Dammeyer JA. Teaching by the nurse: how important is it to patients? Appl Nurs Res: ANR. 2001;14(1):11-17.

19. McCauley J, Jenckes MW, Tarpley MJ, Koenig HG, Yanek LR, Becker DM. Spiritual beliefs and barriers among managed care practitioners. $J$ Relig Health. 2005;44(2):137-146.

20. Kalantari SHKM, Abbaszadeh A, Sanagoo A, Borhani F. Nurses' perception of performance of patient education. Jentashapir Q J Student. 2011;2(4):167-174.

21. M H. Viewpoints of employed nurses in hospitals of Birjand city regarding existing barriers in patient education. Mod Care Sci Q Birjand Nurs Midwifery Facul. 2011;8(3):152-158. 